\title{
State-of-the-Art in Integrated Optical Microspectrometers
}

\author{
Reinoud F. Wolffenbuttel
}

\begin{abstract}
Microspectrometers fabricated in silicon using microelectromechanical systems technologies are versatile microinstruments: small, lightweight, and featuring a demonstrated capability for spectral analysis. When realized using silicon process compatible technologies, low-cost batch fabrication of an intelligent optoelectronic system-on-a-chip is feasible by cointegration of optics with microelectronic circuits. However, the spectral resolution of devices presented so far has been limited to about $R=15$, which does restrict application. This paper provides an overview of microspectrometers operating in the visible and infrared spectral range. Moreover, it is demonstrated that the resolution is primarily limited due to the short optical path that is inherent to a microsystem, optical properties of silicon IC-process compatible materials, and lack of adequate optical signal conditioning.
\end{abstract}

Index Terms-Integrated silicon microsystem, microelectromechanical systems (MEMS), optical sensor, spectrometer.

\section{INTRODUCTION}

$\mathbf{S}$ PECTRAL analysis of an optical spectrum is a well-established technique in physics, chemistry, and biology. Spectroscopic measurement of the emission and absorption spectra of a particular atom provides detailed information about its energy band structure, whereas analysis of a molecule shows the energies associated with the chemical bonds [1]. In chemical analysis, the fluorescence spectrum is widely used to identify the composition of a sample solution and to measure their concentrations [2]. Similarly, in chromatography, the wavelength dependent absorption of the chemical constituent between a light source and the entrance slit of a spectrometer is measured in both the visible and IR part of the spectrum [3], [4]. Fluorescence signals are also investigated for monitoring of photosynthesis in vegetation [5]. Based on such measurements, it might be possible to monitor plant condition and use this information for on-line control of illumination conditions in such a way that photosynthesis is maximized, without causing plant stress.

Available high-performance multiple-grating macroscopic spectrometers feature an impressive spectral resolution $R=\lambda / \Delta \lambda$ that exceeds $R=10^{6}$, where $\Delta \lambda$ denotes the $-3 \mathrm{~dB}$ power bandwidth [also referred to as the full-width half-maximum (FWHM)] at a particular wavelength setting $\lambda$ [6]. However, these are bulky and expensive. Such a resolution

Manuscript received March 30, 2003; revised August 19, 2003. This work was supported in part by the STW Technology Foundation, The Netherlands, under Grant DEL.3733.

The author is with the Department of Microelectronics, Faculty of Information Technology and Systems, Delft University of Technology, Delft, The Netherland (e-mail: R.F.Wolffenbuttel@ITS.TUDelft.NL).

Digital Object Identifier 10.1109/TIM.2003.821490 specification is required in, e.g., astronomy, but often exceeds by far what is required in industrial applications, where issues such as costs, sample volume to be chemically analyzed, and measurement time prevail. Microspectrometers satisfy these additional requirements better. These are small, lightweight, and some are fabricated using silicon process compatible technologies, thus featuring the possibility for realizing an intelligent optoelectronic system-on-a-chip by cointegration of optics with microelectronics [7]. However, the primary performance parameter, the spectral resolution, is limited to $R=15$.

First, an overview of state-of-the-art microspectrometers in silicon is given, followed by a discussion on the limitations imposed by the dimensions or the fabrication techniques on the spectral resolution in such a device. Finally, directions for further research are given.

\section{INTEGRATED MICROSPECTROMETERS}

Spectrometers are basically composed of the following components:

- an input slit for spatial sampling of the radiation;

- a collimating lens (mirror) to produce a collimated light beam;

- a dispersion element (in case of a grating, it deflects the different wavelengths presented in the light beam to different angles);

- a focusing lens that produces image of the input slit in the plane of the sensor with the position of the image depending on the wavelength;

- a detector or array of detectors.

The optical path is in between, as shown in Fig. 1.

Adverse effects of scaling on each of these components should be considered when scaling the dimensions of a spectrometer down to the microsystem level.

A first attempt toward the realization of a miniaturized spectrometer is the planar waveguide spectrometer presented by Goldman et al. [8]. The device is used for chemical analysis and is composed of a waveguide on top of a glass substrate, as shown in Fig. 2. Two buried gratings are etched in the substrate before deposition of the waveguide material and are used for coupling light in and out of the waveguide. While travelling through the waveguide the light interacts with the chemical sample. The resulting spectrum is dispersed at the outcoupling grating. An array of photodiodes is used to analyze the resulting spatially separated spectrum. An IC-process 


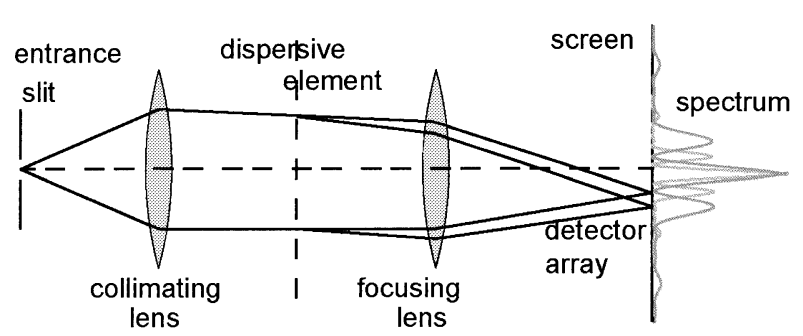

Fig. 1. Simplified schematic of the basic spectrograph in case of a grating and with two spectral components separated.

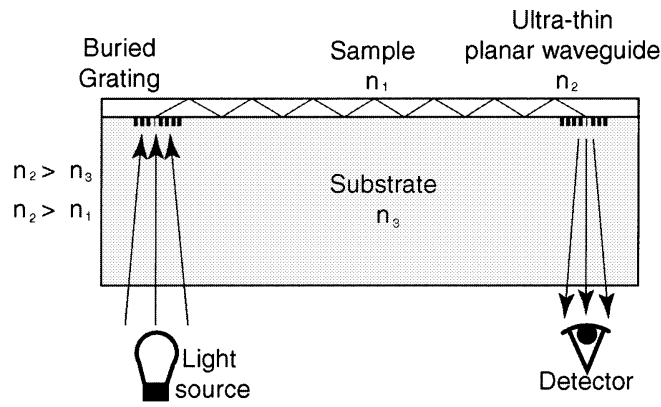

Fig. 2. Waveguide-based spectrometer for analysis of a sample fluid with index of refraction $\mathrm{n}_{1}$ [8].

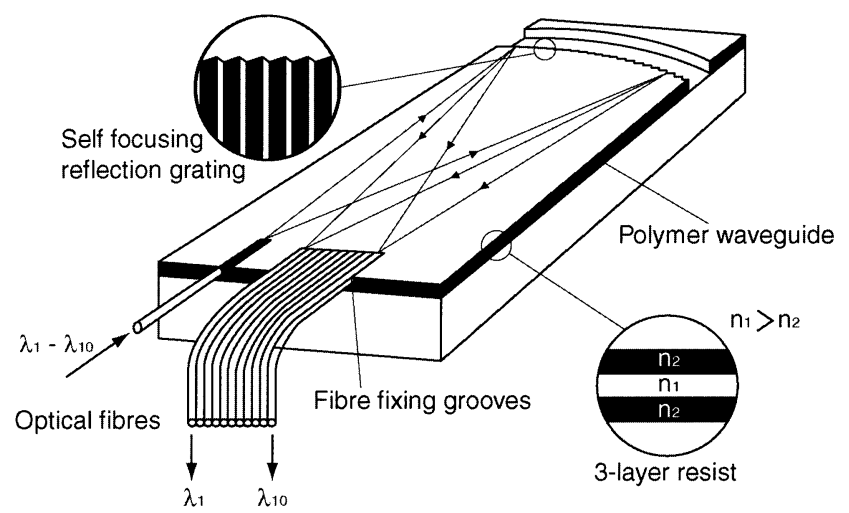

Fig. 3. Waveguide-based spectrometer with fibers for in- and outcoupling of light [11].

compatible implementation of this principle has yielded an impressive resolution of $\Delta \mathrm{n}=4.10^{-6}$ [9], [10].

Fiber technology and silicon micromachining are combined to yield the more universally applicable planar waveguide-based grating spectrometer shown in Fig. 3 [11]. The light to be analyzed is introduced into the polymer waveguide through an optical fiber, dispersed in a reflection grating, that has been fabricated using deep reactive ion etching of the polymer, and projected onto an array of fibers that guide the spectral components to an array of photodetectors. The device area is $18 \times 6.4 \mathrm{~mm}^{2}$ and the resolution obtained is $R=10$ over a spectral range extending between 720 and $900 \mathrm{~nm}$. It is interesting to note that this microspectrometer has so far been the only type that has become commercially available so far [12].

This approach has also been implemented using a silicon-oxi-nitride ( $\mathrm{SiON}$ ) layer as the waveguide. The properties of a waveguide enable the design of a self-focussing transmission grating. A FWHM of $9 \mathrm{~nm}$ has been reported at $450 \mathrm{~nm}$; thus, $\mathrm{R}$ is about 50 [13].

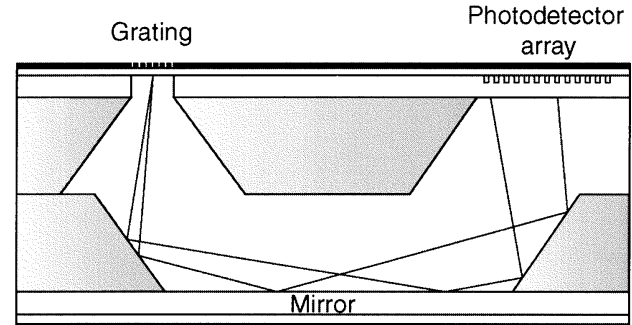

Fig. 4. Micromachined grating-based microspectrometer in silicon for operation in the visible/near IR part of the spectrum [18].

Microelectromechanical systems (MEMS) technology has been used for fabrication of a Michelson interferometer with one electrostatically actuated mirror using a comb drive. A resolution $R=100$ at $633 \mathrm{~nm}$ was reported [14]. Micromachined gratings have also been combined with a CCD camera in a hybrid system for operation as a spectrometer [15]. A resolution $R=70$ at $633 \mathrm{~nm}$ was reported, which is due to the relatively large distance between grating and camera.

These devices are not directly silicon process compatible, and the spectral resolution limit application to the areas of consumer products and quality inspection. However, these typically require the extremely low-cost in high volume fabrication that silicon IC technology can offer. Thus, research has been directed toward integrated silicon devices.

\section{INTEGRATED SILICON MicROSPECTROMETERS}

\section{A. Fabrication of Optical Components}

Gratings and Fabry-Perot resonance cavities have been fabricated in silicon and have been operated successfully as dispersion elements in a microspectrometer. Also, the fabrication of arrays of integrated silicon photon detectors (in case of operation in the visible spectral range) and thermoelectric detectors (in IR applications) has been demonstrated [16]-[32] .

\section{B. Grating-Based Microspectrometers}

Silicon bulk micromachining techniques have been employed for the fabrication of an integrated grating plus detector array in silicon for operation as microspectrometer in the visible and near-infrared spectral range [18]. It is composed of two silicon $p$-type wafers on which an $n$-doped epitaxial layer is grown and an oxide plus nitride layer is deposited. On the other wafer, a metal layer is deposited to form a mirror. In one layer an array of pn junctions is fabricated and the metal is used for fabrication of the grating and for preventing direct illumination of the photodiodes. Subsequently, electrochemically-controlled etching (ECE) is applied to construct channels that form, after wafer bonding, an optical path between a grating and an array of photodiodes, as shown in Fig. 4.

An electrically isolated region of epilayer is formed by deep boron diffusions to define the locations of the grating and backside-illuminated photodiodes. A 32-element $4-\mu \mathrm{m}$ pitch symmetrical (2- $\mu \mathrm{m}$ line $/ 2-\mu \mathrm{m}$ space) grating is used and $2-\mu \mathrm{m}$ wide photodiodes with $4-\mu \mathrm{m}$ pitch are formed by implantation into the epilayer. The aluminum, that is conventionally exclusively used for interconnect, is also used for fabrication of the grating and for shielding the array of photodiodes 


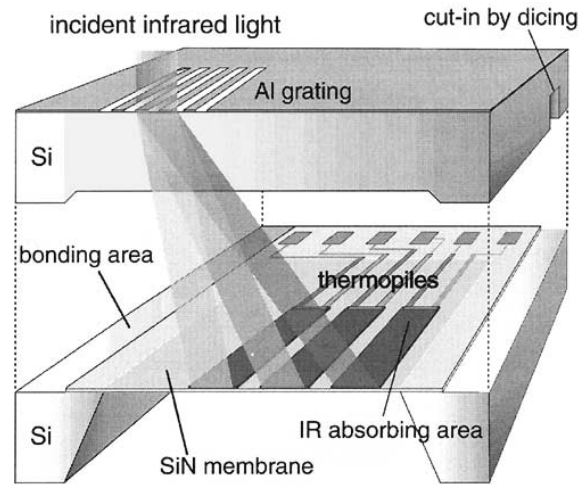

Fig. 5. Schematic view of the grating-based microspectrometer for IR [21].

to prevent frontside illumination by the incident light. The light that is dispersed in the grating is projected onto a bevel micromachined in a $\langle 100\rangle$ wafer. The slope of the bevel has a well-defined angle of $54.7^{\circ}$ that originates from the intersection between the $\langle 100\rangle$ wafer plane and the $\{111\}$ etch bordering planes after anisotropic etching. After reflection on the epilayer of the lower wafer and a second bevel, the light is projected on the array of photodiodes.

A major problem in this device is the roughness of the bulk micromachined surfaces, which results in scattering of the reflected light. As the dispersed light is reflected three times before being projected on the photodiode array, a surface roughness comparable to that of the polished frontside of a wafer is required. This was achieved by avoiding the high temperature steps that are used for epilayer growth and the deep boron diffusion and by applying an oxide reflow step [19]. The functional separation of the device into an active wafer with photodiodes and circuits and a passive reflection wafer, with low-temperature wafer bonding at the very last processing step circumvents any fabrication compatibility infringement and is a huge advantage [20]. A resolution $R>10$ has been achieved at $\lambda=600 \mathrm{~nm}$.

Microspectrometers have also been developed for operation in various wavelength ranges in the IR spectral range. Bulk silicon and aluminum are used for the optical path and the multiple-slit grating, respectively. Silicon is highly transparent for wavelengths, exceeding $1 \mu \mathrm{m}$, beyond which free-carrier absorption can be disregarded. Therefore, the bulk silicon can be used to define the optical path rather than air. Also, in the IR spectral range, aluminum can be used for the fabrication of the grating. The IR spectrometer, shown schematically in Fig. 5, consists of two independently processed wafers, which are bonded in the final step [21]. Similar to the spectrometer operating in the visible spectral range, a two-wafer approach is taken. Thermal detectors are used, as the silicon bandgap does not allow for photon detection in the IR spectral range.

The grating is composed of 30 or 60 slits with a grating constant ranging from 4 to $20 \mu \mathrm{m}$. The length of the strips is $400 \mu \mathrm{m}$. The width of a single IR detector on the bottom wafer is $100 \mu \mathrm{m}$. The number of detectors in one array ranges between 6 and 16 and depends on the detectable wavelength range, which is, in turn, determined by the grating constant.

Fig. 6 shows the top view of a fabricated device. The upper half shows the detector array.

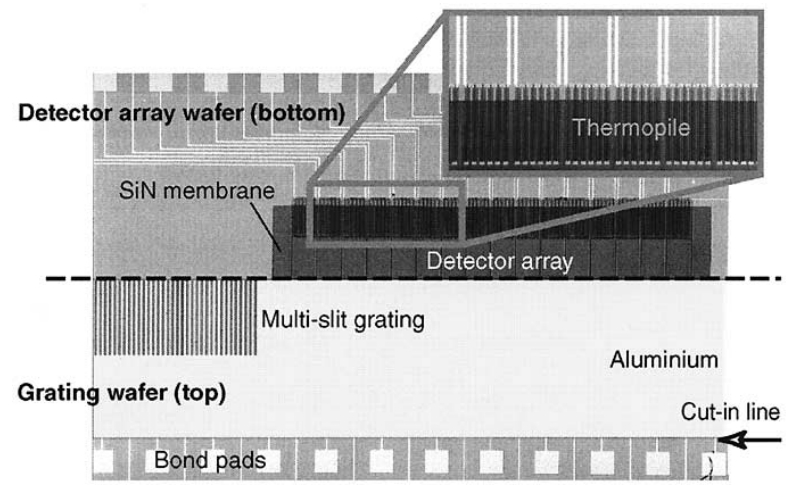

Fig. 6. Microfabricated IR microspectrometer in silicon [21]

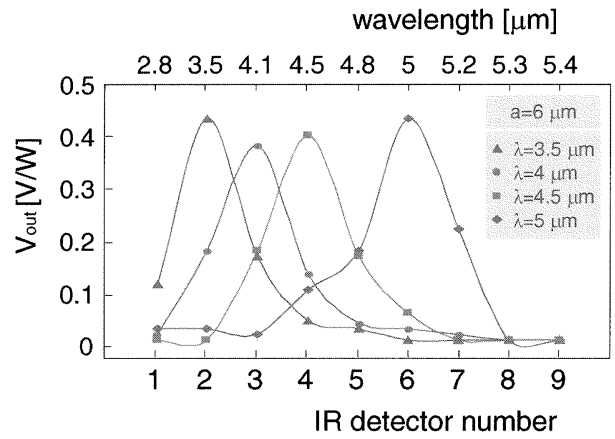

Fig. 7. Spectral response of the IR microspectrometer with grating constant at $6 \mu \mathrm{m}$ when illuminated with a monochromator illuminator set at $\lambda=3.5 \mu \mathrm{m}$, $4 \mu \mathrm{m}, 4.5 \mu \mathrm{m}$, and $5 \mu \mathrm{m}$.

The thermocouple responses at a 6- $\mu \mathrm{m}$ grating constant for a number of monochromatic components are shown in Fig. 7. The response peaks at a larger detector number in case of longer wavelength illumination. The FWHM at $\lambda=5 \mu \mathrm{m}$ is about 0.5 $\mu \mathrm{m}$, thus $\mathrm{R}$ is about 10 .

\section{Fabry-Perot-Based Microspectrometers}

A number of principles based on optical resonance and suitable for the fabrication of microspectrometers are available (Michelson, Mach-Zehnder, Sagnac, and Fabry-Perot). Whereas Mach-Zehnder-based systems have found widespread application in integrated waveguides, Fabry-Perot based cavities are used in free-field microspectrometers.

The basic Fabry-Perot interferometer is composed of two highly-reflective and parallel mirrors spaced a well-defined distance $d_{F P}$ apart with the reflecting part facing each other. The space in between the mirror surfaces is essentially a resonance cavity. The operation is similar to the grating in the sense that traversal leads to a phase difference $\varphi=\mathrm{k}_{\mathrm{o}} 2 \mathrm{nd}_{\mathrm{FP}}$, with $\mathrm{k}_{\mathrm{o}}$ the free-space wave vector Interference of these components results in a wavelength dependent transmission described by the Airy transmission coefficient [22], [23]. As the detector is placed directly under the resonator, no focussing optics is required. Resonance takes place at $2 \mathrm{nd}_{\mathrm{FP}}=m \lambda_{\mathrm{o}}$, with $m$ an integer number. The spectral selectivity is expressed in terms of the intrinsic finesse $\mathrm{F}_{\text {int }}$ of the resonator. In the case of two perfectly parallel mirrors, $\mathrm{F}_{\text {int }}=\pi \sqrt{ } \mathrm{r} /(1-\mathrm{r})$, where $r$ denotes the amplitude reflection coefficient of the mirror. 


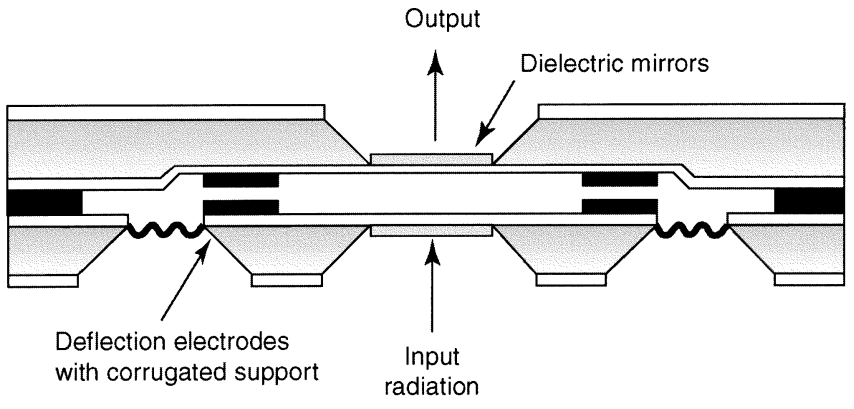

Fig. 8. Microfabricated Fabry-Perot-based microspectrometer in silicon [24].

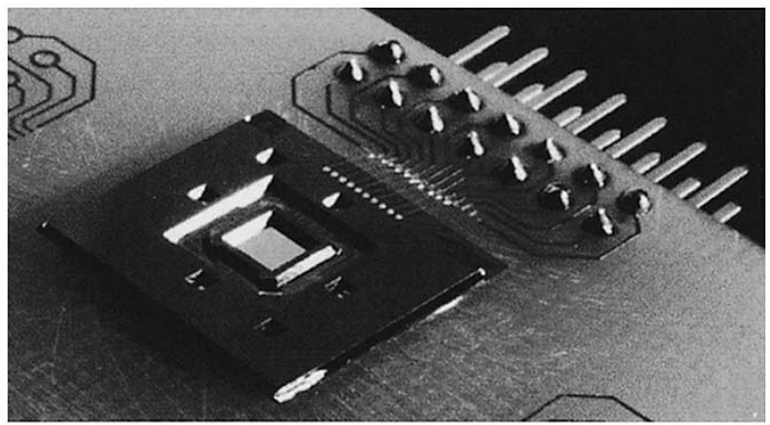

Fig. 9. Photograph of the two-wafer bulk-micromachined Fabry-Perot interferometer. A frame is used to keep the membrane flat [28].

The simplest realization of the Fabry-Perot based microspectrometer uses bulk micromachining on two wafers with subsequent wafer-to-wafer bonding [24]-[30]. Fig. 8 shows the basic device structure. Applying a voltage makes it possible to tune the resonance cavity width to the desired wavelength. In the improved version shown in Fig. 9, a surrounding silicon frame is used to ensure a flat membrane at the mirror area. The deformation of the membrane due to an electrostatic force acting on it is in the membrane area outside the frame [28].

This technique prevents a reduced optical performance that results from the curvature of a simple suspended membrane with a mirror surface, at the expense of a higher voltage required for yielding a certain deflection at the same membrane area. Beam suspension [27] and corrugations [29] are also used for this purpose.

The main challenges in the design of this type of Fabry-Perot interferometer are

- the high voltage required to tune the movable mirror over a sufficiently large spectral range;

- the fabrication of mirror surfaces of sufficient reflectivity and flatness;

- to achieve parallelism between the two mirrors.

An alternative approach that circumvents these problems uses 16 Fabry-Perot resonators of different fixed cavity spacing. Oxide layers are used to space the mirrors, thus ensuring parallel mirrors. Four subsequent masked oxide etch steps are applied to fabricate cavities of 16 different thickness. The 16 channels are designed to cover the entire visible spectral range. The fabrication of these Fabry-Perot resonators is compatible with a standard CMOS process, thus enabling the on-chip integration of circuits for selection and readout of the array photodiodes covered by the different resonators. The

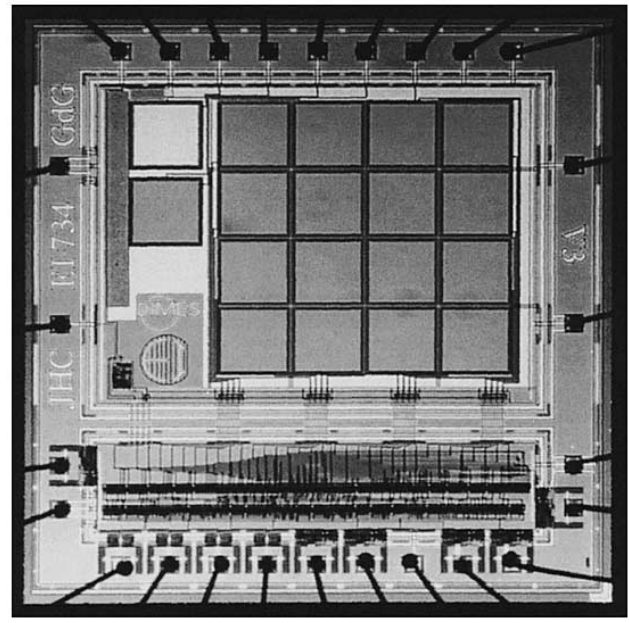

Fig. 10. CMOS multichannel microspectrometer with 16 Fabry-Perot resonance elements on top of a chip containing a photodiode array and circuits for readout and interfacing a serial bus [31].

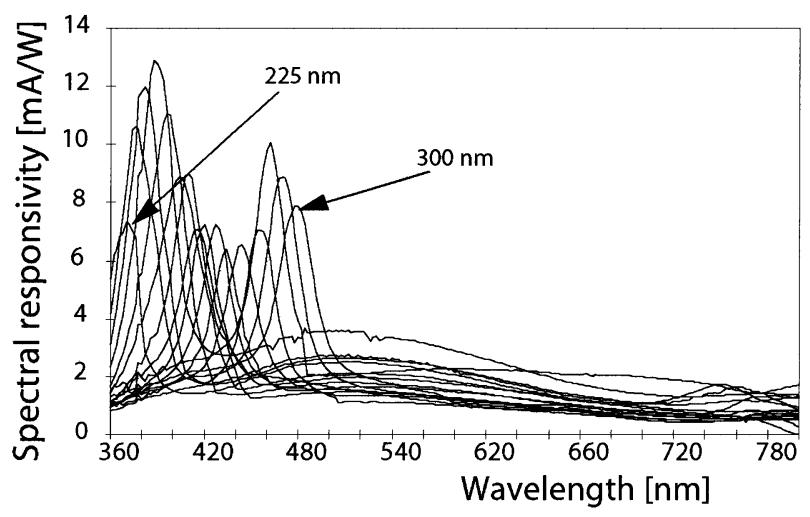

Fig. 11. Spectral responses of the 16 Fabry-Perot channels with oxide spacer thickness varying between $225 \mathrm{~nm}$ and $300 \mathrm{~nm}$.

resulting device is shown in Fig. 10 [31]. An additional channel is used for the compensation of background and scattered light. The latter is due to roughness of the mirror material [32]. This device contains no moving parts. A major disadvantage is the inefficient use of impinging optical power. The light is projected, and thus distributed over the array before dispersion, thus each etalon receives only the part of the power proportional to the number of elements.

Fig. 11 shows the results. The resolution is intrinsically limited to $R=16$ and even lower as is demonstrated by the overlapping response curves. It should be noted that these curves also include the wavelength dependence of the sensitivity of the integrated photodiodes. The attenuation at resonance is significant due to absorption of light of resonance frequency in the mirrors.

\section{SPECTRAL RESOLUTION}

\section{A. Limitations in Grating-Based Microspectrometers}

The spectral resolution of the microspectrometers presented is limited to about $R=15$. The main impediment for a fully integrated silicon optical microspectrometer is the definition of a sufficiently long optical path and the implementation of lenses 
or deformed mirrors. The reasons are not the same for the different devices presented and primarily depend on whether the spectrum is dispersed by a grating or a resonance cavity.

The spectral resolution in a grating-based spectrometer, when using the first-order diffraction spectrum, is in principle equal to the number of slits, $\mathrm{N}$, in the grating: $R=\mathrm{N}$ [33]. However, this assumes Fraunhofer rather than Fresnel diffraction. For ensuring Fraunhofer diffraction (the laws of far field optics to be valid) the wavefront of both the radiation impinging on the grating and on the detector should be plane. This implies that the distance between the entrance slit and the grating, as well as the distance between the grating (which can within this context also be considered a single slit) and the surface onto which the dispersed spectrum is projected (i.e., the detector) should be "large enough." This condition is more quantitatively expressed as the Rayleigh distance, $\mathrm{D}=\mathrm{w}^{2} / \lambda$, where $\mathrm{w}$ denotes the slit width and $\lambda$ the wavelength of the light incident on the slit.

Obviously, a parallel beam at minimum optical path length can be obtained using collimating optics at the entrance of the spectrometer and focussing of the dispersed spectrum leaving the grating. The collimating lens at the entrance can be incorporated in the package, but this has so far not been demonstrated. Fabrication of focusing optics in between the grating and the detector is a technological hurdle that has so far not been taken. The consequence for devices realized is a grating without optical signal conditioning on either side. This directly determines the minimum optical path required and thus the dimensions of the device.

In the case of an $N$-element grating with a pitch (= grating constant) $a$, i.e., a regular pattern of $a / 2$ wide aluminum strips spaced at $a / 2$, results in a "slit" of width $\mathrm{w}=\mathrm{N} a$. The resulting Rayleigh distance at wavelength $\lambda$ is equal to $\mathrm{D}=\mathrm{N}^{2} a^{2} / \lambda$. The length of optical path available is in this device equal to the wafer thickness $\mathrm{t}_{\mathrm{w}}=525 \mu \mathrm{m}$. Given this constraint, $\mathrm{N}_{\max }^{2}=$ $\mathrm{t}_{\mathrm{w}} \lambda / \mathrm{a}^{2}$. The pitch is limited by technological constraints and the by diffraction angle that results from the maximum wavelength to be analyzed. For the grating based microspectrometer operating in the visible spectral range, depicted in Fig. 4, $\mathrm{N}_{\max }$ follows for $a=4 \mu \mathrm{m}$ and $\lambda=500 \mathrm{~nm}$ as $\mathrm{N}_{\max }=13$. A similar calculation holds for the IR spectrometer depicted in Fig. 5. Using $a=4 \mu \mathrm{m}$ and $\lambda=4 \mu \mathrm{m}$ yields $\mathrm{N}_{\max }=12$. The measured performance shown in Fig. 7 is in good agreement with this theoretical limit. It would be pointless to increase the number of elements in the grating at the given device dimensions.

An improved resolution is feasible only by having a larger $N$ associated with an enlarged optical path length between grating and detector array. This can be achieved by, e.g., stacking of wafers. Also, the pitch can be further reduced. An optical path exceeding $50 \mathrm{~mm}$ can be realized on a $10 \times 10 \mathrm{~mm}^{2}$ chip using mirrors. In the case of a grating constant $a=1 \mu \mathrm{m}$, a resolution at $\lambda=600 \mathrm{~nm}$ of $R=(\lambda \mathrm{L})^{0.5} / a=170$ would be possible. Such a selectivity is adequate in many of the applications mentioned.

\section{B. Limitations in Fabry-Perot-Based Microspectrometers}

The constraints that limit the spectral resolution in a Fabry-Perot microspectrometers are different from those in grating based systems. The resolution is described by $R=m \mathrm{~F}_{\text {int }}$. Fabry-Perot resonators in microspectrometers are usually operated in the first-order mode $(m=1)$ with the resolution equal to the Finesse $\mathrm{F}_{\text {int }}=\pi \sqrt{ } \mathrm{r} /(1-\mathrm{r})$. Thus, a high resolution implies a high reflectance of the mirrors. However, in addition, a high transmission at resonance is desirable. The problem is that a minimum mirror thickness is required for a metal film based mirror (typically $>15 \mathrm{~nm}$ for $r>0.90$ [34]) and absorption increases rapidly with metal layer thickness (maximum transmission at resonance is limited to $\mathrm{T}_{\max }=15 \%$ due to absorption in a 15 -nm thick metal layer).

Thus, the resolution is limited to $F_{\text {int }}=\pi \sqrt{ } 0.9 /(0.1)=30$, which is a factor of 3 better than the results shown in Fig. 11. The low throughput at resonance results in a poor signal-to-noise ratio (SNR). High reflectance and low absorption can be combined in a multiple-layer dielectric mirror [35]; however, the fabrication of such a stack of alternating materials is marginally compatible with silicon microelectronic processes.

Similar to macroscopic devices, the spectral resolution can be significantly improved by operating the Fabry-Perot resonator at a higher-order mode. However, this solution suffers from two complications. First, the nominal cavity width required becomes incompatible with what can be realized using conventional micromachining techniques $(R=1000$ at $600 \mathrm{~nm}$ would require $m=73$ and a cavity width $\left.\mathrm{d}_{\mathrm{FP}}=44 \mu \mathrm{m}\right)$. Second, the spectral resolution would become primarily limited by the parallelism of the two mirrors. Changes in the cavity width within one device obviously yield a range of resonance frequencies and thus of a widening of the overall resonance. This effect can be analytically expressed in the effective finesse: $1 / \mathrm{F}_{\text {eff }}^{2}=$ $1 / F_{\text {int }}^{2}+1 / F_{D}^{2}$, where $F_{\text {int }}$ denotes the intrinsic finesse discussed before, which depends on the material property $r$, and $\mathrm{F}_{\mathrm{D}}$ the defect finesse, which is limited by system defects such as nonperfect parallelism [30]. Multiple-location servo control of cavity spacing based on capacitive displacement sensing and electrostatic actuation has been applied to optimize parallelism [36]; however, $\mathrm{F}_{\text {eff }}$ in micromachined silicon devices are typically limited to $\mathrm{F}_{\text {eff }}=50$ [30]. The defect finesse is also the reason for the much lower resolution shown in Fig. 11 compared to the theoretical value $R=30$. The roughness of the deposited layer used for the mirror results in a low $\mathrm{F}_{\mathrm{D}}$, thus limiting the spectral resolution.

Finally, the free spectral range (FSR) is in principle limited to avoid overlap between the shortest wavelength in the first-order mode and the longest wavelength in the subsequent secondorder mode: FSR $=\lambda^{2} / 2 \mathrm{~d}_{\mathrm{FP}}$. However, practical cavity width modulation using electrostatic actuation limits $\Delta \mathrm{d}_{\mathrm{FP}} / \mathrm{d}_{\mathrm{FP}}$ to about $30 \%$ due to pull-in of the microstructure.

These limitations, in addition to the absorption in metal mirrors mentioned already, are generic to a resonator-based device with an air cavity.

\section{CONCLUSION}

The optical performance of grating-based microspectrometers does not scale well with reducing device dimensions. The resolution is typically limited to the range $10<R<50$. This value can be improved by an order of magnitude using on-chip mirrors. However, if one decides to implement mirrors, there would be good reason to go one step further and actually implement adequate signal conditioning using deformable mirrors 
for collimation and focusing [37]. This would improve resolution by another order of magnitude, similar to [38]. The resulting selectivity would be adequate in many of the applications mentioned.

In Fabry-Perot based devices, the performance restricted to $R$ is about 50 , primarily due to optical limitations of the materials used for the mirrors, as is expressed in the defect finesse and the low optical transmission at resonance. This is not a major problem in channel switching in optical communication, but it is an impediment in a microspectrometer.

\section{ACKNOWLEDGMENT}

The author would like to thank the Netherlands Technology Foundation (STW), DIMES Technology Center, for the fabrication of devices, and former students T. A. Kwa, J. H. G. Correia, and S.-H. Kong for their contributions in the research on integrated silicon optical microspectrometers.

\section{REFERENCES}

[1] B. Stuart, Modern IR Spectroscopy. New York: Wiley, 1996.

[2] V. Iordanov, G. Lubking, R. Ishihara, R. F. Wolffenbuttel, P. M. Sarro, and M. J. Vellekoop, "Silicon thin-film UV filter for NADH analysis," Sens. Actuators A, pp. 161-166, 2002.

[3] D. Rossberg, "Silicon micromachined infrared sensor with tunable wavelength selectivity for application in infrared spectroscopy," Sens. Actuators A, vol. 46-47, pp. 413-416, 1995.

[4] G. Minas, J. S. Martins, C. Pereira, C. Lima, R. F. Wolffenbuttel, and J. H. Correia, "Lab-on-a-chip for measuring uric acid in biological fluids," in Proc. Eurosensors XVI, Prague, Czech Republic, Sept. 15-18, 2002, pp. 66-69.

[5] A. Rosema, "Potential of chlorophyll fluorescence for remote sensing of canopy photosynthesis," in Proc. OECD Workshop on Remote Sensing for Agriculture For the Environment, Kiffisia, Greece, Sept. 17-20, 2002.

[6] P. Lindblom, M. Meinander, and T. Olsson, "Spectroscopy with the MEGA spectrometer, a very high resolution grating spectrometer," Rev. Sci. Instrum., vol. 61, pp. 2546-2548, 1990.

[7] R. F. Wolffenbuttel, "Silicon photodetectors with a selective spectral response," in Sensors Update, H. Baltes, J. Hesse, and J. Korvink, Eds. Berlin, Germany: Wiley-VCH, 2001, vol. 9, pp. 69-101.

[8] D. Goldman, P. White, and N. Anheier, "Miniaturised spectrometer employing planar waveguides and grating couplers for chemical analysis," Appl. Opt., vol. 29, pp. 4583-4589, 1990.

[9] P. V. Lambeck, "Integrated chemo-optical sensors," Sensors and Actuators, vol. B8, pp. 103-116, 1993.

[10] R. G. Heideman, R. P. H. Kooyman, and J. Greve, "Performance of a highly sensitive optical waveguide Mach-Zehnder interferometer immunosensor," Sens. Actuators B, vol. 10, pp. 209-217, 1993.

[11] J. Mohr, B. Anderer, and W. Ehrfeld, "Fabrication of a planar grating spectrograph by deep-etch lithography with synchrotron radiation," Sens. Actuators A, vol. 25-27, pp. 571-575, 1991.

[12] [Online]. Available: www.getspec.com

[13] D. Sander and J. Müller, "Self-focussing phase transmission grating for an integrated optical spectrometer," Sens. Actuators A, vol. 88, pp. 1-9, 2001.

[14] O. Manzardo, H. P. Herzig, C. R. Marxer, and N. F. de Rooij, "Miniaturized time-scanning Fourier transform spectrometer based on silicon technology," Opt. Lett., vol. 24, pp. 1705-1707, 1999.

[15] G. M. Yee, N. I. Maluf, P. A. Hing, M. Albin, and G. T. A. Kovacs, "Miniature spectrometers for biochemical analysis," Sens. Actuators A, vol. 58, pp. 61-66, 1997.

[16] K. Hirabayashi, H. Tsuda, and T. Kurokawa, "Narrow-band tunable wavelength selective filters for Fabry-Perot interferometers with a liquid crystal intercavity," IEEE Photon. Technol. Lett., vol. 3, pp. 213-215, 1991

[17] J. W. Holm-Kennedy, K. W. Tsang, W. W. Sze, F. Jiang, and D. Yang, "A novel monolithic chip-integrated color spectrometer: The distributed wavelength filter component," Proc. SPIE, vol. 1527, pp. 322-331, 1991.

[18] T. A. Kwa and R. F. Wolffenbuttel, "Integrated grating/detector array fabricated in silicon using micromachining techniques," Sens. Actuators A, vol. 31, pp. 259-266, 1992.
[19] T. A. Kwa, P. J. French, R. F. Wolffenbuttel, P. M. Sarro, L. Hellemans, and J. Snauwaert, "Anisotropically etched silicon mirrors for optical sensor applications,” J. Electrochem. Soc., vol. 142, pp. 1226-1233, 1995.

[20] R. F. Wolffenbuttel, Ed., Silicon Sensors And Circuits: On-Chip Compatibility. London, U.K.: Chapman \& Hall, 1996.

[21] S.-H. Kong, D. D. L. Wijngaards, and R. F. Wolffenbuttel, "Integrated microspectrometer based on a diffraction grating," Sens. Actuators A, vol. 92, pp. 88-95, 2001.

[22] B. Saleh and M. C. Teich, Fundamentals of Photonics. New York: Wiley, 1991.

[23] J. M. Vaughan, The Fabry-Perot Interferometer: History, Theory, Practice, and Applications. London, U.K.: Adam Hilger, 1989.

[24] N. F. Raley, D. R. Ciarlo, J. C. Koo, B. Beiriger, J. Trujillo, C. Yu, G. Loomis, and R. Chow, "A Fabry-Perot microinterferometer for visible wavelengths," in Proc. IEEE Workshop MEMS, Travemünde, Germany, Feb. 4-7, 1992, pp. 170-173.

[25] A. T. T. D. Tran, Y. H. Lo, Z. H. Zhu, D. Haronian, and E. Mozdy, "Surface-micromachined Fabry-Perot tunable filter," IEEE Photon. Technol. Lett., vol. 8-3, pp. 393-395, 1996.

[26] P. M. Zavracky, K. L. Denis, H. K. Xie, T. Webster, and P. Kelley, "A micromachined scanning Fabry-Perot interferometer," in Proc. SPIE Micromachined Devices and Components IV, Santa Clara, CA, 1998, pp. 178-187.

[27] K. Aratani, P. J. French, P. M. Sarro, D. P. Poenar, and R. F. Wolffenbuttel, "Surface micromachined tunable interferometer array," Sens. Actuators A, vol. 41-42, pp. 17-23, 1994.

[28] J. H. Correia, M. Bartek, and R. F. Wolffenbuttel, "Bulk micromachined tunable Fabry-Perot micro-interferometer for the visible spectral range," Sens. Actuators A, vol. 76, pp. 191-196, 1999.

[29] _ - "High-selectivity single-chip spectrometer in silicon for operation in the visible part of the spectrum," IEEE Trans. Electron Devices, vol. 47, pp. 553-559, Mar. 2000.

[30] J. H. Jerman, D. J. Clift, and S. R. Mallinson, "A miniature Fabry-Perot interferometer with a corrugated silicon diaphragm support," Sens. Actuators A, vol. 29, pp. 151-158, 1991.

[31] J. H. Correia, G. de Graaf, S.-H. Kong, M. Bartek, and R. F. Wolffenbuttel, "Single-chip CMOS optical micro-interferometer," Sens. Actuators A, vol. 82, pp. 191-197, 2000.

[32] J. H. Correia, G. de Graaf, M. Bartek, and R. F.Wolffenbuttel, "A CMOS optical microspectrometer with light-to-frequency converter, bus interface and stray-light compensation," IEEE Trans. Instrum. Meas., pp. 1530-1537, Dec. 2001

[33] M. Born and E. Wolf, Principles of Optics, 5th ed. New York: Pergamon, 1975.

[34] M. A. Ordal, "Optical properties of the metals $\mathrm{Al}, \mathrm{Co}, \mathrm{Cu}, \mathrm{Au}, \mathrm{Fe}, \mathrm{Pb}$, $\mathrm{Ni}, \mathrm{Pd}, \mathrm{Pt}, \mathrm{Ag}, \mathrm{Ti}$, and $\mathrm{W}$ in the infrared and far infrared," Appl. Opt., vol. 22, no. 7, pp. 1099-1119, 1983.

[35] H. A. Macleod, Thin-Film Optical Filters, 2nd ed. London, U.K.: Adam Hilger, 1986.

[36] T. R. Hicks, N. K. Raey, and P. D. Atherton, "The application of capacitance micrometry to the control of Fabry-Perot etalons," J. Phys. E, Sci. Instrum., vol. 17, pp. 49-54, 1984.

[37] G. V. Vdovin, O. Akhzar-Mmehr, P. M. Sarro, D. W. de Lima Monteiro, and M. Loktev, "Arrays of spherical micromirrors and molded micolenses fabricated with bulk Si micromachining," Proc. SPIE, vol. 4945, pp. 107-111, 2003.

[38] D. Sander and J. Müller, "Self-focusing phase transmission grating for an integrated optical spectrometer," in Sens. Actuators A, vol. 88, 2001, pp. 1-9.

Reinoud F. Wolffenbuttel received the M.Sc. and Ph.D. degrees from the Delft University of Technology (DUT), Delft, The Netherlands, in 1984 and 1988, respectively.

Between 1986 and 1993, he was an Assistant Professor and since 1993, he has been an Associate Professor with the Department of Microelectronics, Faculty of Information Technology and Systems, DUT, and is involved in instrumentation and measurement in general and on-chip functional integration of microelectronic circuits and silicon sensors, IC process compatible MEMS fabrication, and silicon microsystems, in particular. He was a Visitor at the University of Michigan, Ann Arbor, in 1992, 1999, and 2001, Tohoku University, Sendai, Japan, in 1995, and EPFL, Lausanne, Switzerland, in 1997.

Dr. Wolffenbuttel is the recipient of a 1997 NWO pioneer award. He served as the General Chairman of the Dutch National Sensor Conference in 1996, Eurosensors in 1999, and the MicroMechanics Europe Workshop in 2003. 